\title{
Editorial: Littoral 2008
}

\author{
Pierpaolo Campostrini • Patrizia Torricelli • \\ David R. Green
}

Received: 8 August 2011 /Revised: 9 August 2011 /Accepted: 10 August 2011 / Published online: 20 August 2011

(C) Springer Science+Business Media B.V. 2011

The Littoral series of conference is a major European event which attracts an international audience of coastal researchers and managers, and public administrations

Littoral 2008 "A changing coast: challenge for the environmental policies", held in Venice, Scuola Grande di San Giovanni Evangelista, in November 2008, was organized by CORILA and by The Coastal \& Marine Union (EUCC). It had about 250 contributions from all over Europe and beyond. A number of workshop and meetings completed the programme, offering a large and comprehensive occasion for gathering and discussion. In fact, the main aim of the conference was to promote a real crossfertilisation between different disciplines and experiences, overcoming the fragmentation that still exists both in coastal policy-making and research.

Littoral 2008 had a unique opportunity to build upon the latest developments in coastal and marine management occurred in 2007-08 in Europe. The evaluation of the EU

\author{
P. Campostrini $(\triangle)$ \\ CORILA, \\ San Marco 2847, \\ 30124 Venice, Italy \\ e-mail: campostrini@corila.it \\ URL: www.corila.it \\ P. Torricelli \\ Environmental Science Department, Università Ca' Foscari, \\ Castello 2737, \\ 30122 Venice, Italy \\ e-mail: torri@unive.it \\ D. R. Green \\ Department of Geography and Environment, Centre for Marine \\ and Coastal Zone Management (CMCZM), \\ University of Aberdeen, \\ St. Mary's, Elphinstone Road, \\ Aberdeen, AB24 UF Scotland, UK
}

ICZM Recommendation (COM(2007)308), showed that while the ICZM approach and principles are valid, there is a further need to support ICZM implementation. In January 2008, the Protocol on ICZM of the Barcelona Convention was signed, representing the first significant step in the development of international legislative instruments for ICZM (the Protocol entered into force on November 2010). The Marine Strategy Framework directive 2008/56/EC was delivered in June 2008 and few days before of the Littoral Conference the European Commission issued the Roadmap for Maritime Spatial Planning (COM (2008) 791 final): this document was actually presented for the first time during the conference by the EC's officers that were present.

The conference was also the final event of the EC-FP6 project ENCORA, that provided a very successful networking structure for creating a new dimension to European cooperation on coastal issues. The coastal portal www. coastalwiki.org developed through ENCORA is still alive, used and updated after 3 years the end of the project: it is an Internet encyclopaedia of more than 1400 high quality Coastal and Marine information pages, made for and by coastal professionals. During Littoral 2008, the ENCORA network was integrated in a new instrument, open to a even wider community: 30 European organisations and networks signed the "Venice declaration" with the intention to set up a Platform, with the aim to enable an informed dialogue with the European Commission and an exchange of information, experience, practices and knowledge between the stakeholder organizations represented in the Platform, which took the name of "Venice Platform" and is still active (www.veniceplatform.eu).

Last but not least, the Littoral 2008 conference was a gathering of scientists and its proceedings are a collection of good-quality scientific papers. In this special issue on 
LITTORAL 2008 conference, five papers have been selected, being representative of the conference programme topics: they have been largely expanded by Authors and peer-reviewed. The papers consider the management of the coastal zone system in all its components, social, economic and environmental, putting in evidence the importance of the integration of scientific disciplines in the costal system assessment.

The conservation of coastal wetlands, lagoons and transitional water systems in a good ecological status is a general and world-wide goal, to be achieved through management policies based on a better understanding of the functioning of these ecosystems. Biodiversity conservation and sustainable development in the coasts are the main general targets of a truly integrated coastal zone management, that need to be made more explicit by the means of reliable, measurable indicators. In addition, safeguarding of the cultural heritage, present along the coasts, from natural and anthropogenic threats, is a rising necessity, to be included in local, national and international policies.

For many years, the use of Decision Support Systems (DSS) to help decision-makers and managers has been promoted, especially for planning and for those decisions implying long-term effects. There is less general understanding about the need to support the science efforts to further improve the state-of-the-art of knowledge, in order to maintain continuously updated models embedded in these DSS. A good policy needs proper governance actions to implement it, as well as adequate monitoring capabilities for assessing the results, feeding-back the suggestions for policy corrections when needed. Co-operation with science is important in all the stages, from the needs' assessment, to the policy building and implementation, up to the monitoring and evaluation stages.

The papers presented here are proof of how much science is necessary and useful, especially when researchers are able to orient their efforts for the solutions of problems felt as urgent by society.

We wish to thank the Authors and the reviewers for their valuable efforts, and the Editor for the continuous support given for the realisation of this special issue. A particular and sincere acknowledgment is due to Job Dronkers (leader of ENCORA) and to Albert Salman (EUCC Director General), for their efforts in the preparation and running of the Littoral 2008 conference. Finally, our gratitude and appreciation goes to Simona Dalla Riva (CORILA), for her precious work in the coordination of this issue. 